\title{
MODELOS DE REFERÊNCIA PARA DESENVOLVIMENTO DE PRODUTOS: CLASSIFICAÇÃO, ANÁLISE E SUGESTÕES PARA PESQUISAS FUTURAS
}

\section{PRODUCT DEVELOPMENT REFERENCE MODELS: CLASSIFICATION, ANALYSIS AND SUGESTIONS FOR FUTURE RESEARCH}

\author{
Eduardo Gomes Salgado*eduardosalgado@unifal-mg.edu.br \\ Valério A. P. Salomon** salomon@feg.unesp.br \\ Carlos Henrique Pereira Mello*** carlos.mello@unifei.edu.br \\ Flávia Duque Marassi Fass**flaviamarassi@hotmail.com \\ Amanda Fernandes Xavier***amandaxavier@unifei.edu.br \\ *Universidade Federal de Alfenas (UNIFAL) \\ **Universidade Estadual Paulista (UNESP) \\ *** Universidade Federal de Itajubá (IEPG/UNIFEI)
}

\begin{abstract}
Resumo: Os modelos de referência para o processo de desenvolvimento de produtos podem contribuir para facilitar a compreensão e a comunicação entre os coordenadores do projeto do produto, para auxiliar nas decisões sobre as funções da gestão do projeto, tais como o planejamento e organização. $O$ presente trabalho tem como objetivo analisar a literatura sobre os modelos para o processo de desenvolvimento de produtos e propor sugestões para pesquisas futuras. O método de pesquisa utilizado foi de cunho conceitual-teórico e o resultado obtido foi a identificação dos setores industriais que não possuem modelos de referência para o processo de desenvolvimento de produtos, tais como o militar, biotecnológico, naval e Arranjos Produtivos Locais, de acordo com a literatura pesquisada. Além disso, o resultado mais significativo obtido pelo estudo é a percepção de que existe uma tendência de desenvolver modelos mais específicos para cada setor industrial. As principais contribuições deste trabalho são mapear os estudos já realizados sobre a adaptação de modelos de referência para o PDP e identificar lacunas para pesquisas futuras nesta área de conhecimento.
\end{abstract}

Palavras-chave: PDP. Modelos de desenvolvimento de produtos. Dimensões do PDP.

\begin{abstract}
The reference models for the product development process can help to facilitate understanding and communication between engineers from product design to assist in decisions about the functions of project management such as planning and organization. This paper aims to analyze the literature on models for the product development process and give suggestions for future research. The research method used was the conceptual-theoretical approach and the result was the identification of industrial sectors that have no role models for the product development process, such as the military, biotechnology, marine and clusters of small firms, according to the literature. Moreover, the most significant outcome from the study is the perception that there is a tendency to develop more specific models for each industrial sector. The main contributions of this work is to map the previous studies on adaptation of reference models for the PDP and identify gaps for future research in this knowledge field.
\end{abstract}

Keywords: PDP. Product development models. PDP dimensions. 


\section{INTRODUÇÃo}

O Processo de Desenvolvimento de Produtos (PDP) refere-se aos passos, atividades, tarefas, estágios e decisões que envolvem o projeto de desenvolvimento de um novo produto ou serviço, ou a melhoria em um já existente, desde a idéia inicial até descontinuação do produto, com a finalidade de sistematizar esse processo. No PDP se identificam os desejos dos clientes, traduzidos em especificações a serem desenvolvidas para gerar soluções técnicas e comerciais. Tudo isso atrelado à estratégia, às restrições, às possibilidades operacionais da empresa e às necessidades dos clientes (BACK, 1983; VINCENT, 1989; CLARK E FUJIMOTO, 1991; ROSENTHAL, 1992; WHEELWRIGHT \& CLARK, 1992; RAMPERSAD, 1995; COOPER \& EDGETT, 1999; PETERS et al., 1999; PAHL et al., 2005; ROZENFELD et al., 2006).

Devido às rápidas mudanças tecnológicas, as empresas estão operando em diversos mercados que exigem uma inovação mais frequente, ciclos de vida de produto mais curtos e um produto com alta qualidade e confiabilidade (NIJSSEN e FRAMBACH, 2000; ILORI, OKE E SANNI, 2000; MARCH-CHORDA et al., 2002; GONZÁLEZ E PALÁCIOS, 2002; MCIVOR e HUMPHREYS, 2004; KOUFTEROS, et al., 2005; XIN, YEUNG e CHENG, 2008). Assim, as pressões geradas pela competição têm levado as organizações a introduzir com mais rapidez os seus produtos no mercado, com menor custo e melhor qualidade (STALK e HOUT, 1990; BLACKBURN, 1991; WHEELWRIGTH e CLARK, 1992; BARNETT e CLARK, 1998; STALK, 1998; OGLIARI, 1999; SILVA, 2001; ROMANO, 2003). A competitividade é fortemente relacionada ao Desenvolvimento de Produtos (DP), embora não seja determinada, exclusivamente, pelo PDP.

O sucesso da organização, frequentemente, depende do período e da consistência no lançamento dos novos produtos. A literatura contém várias prescrições para o DP de alto desempenho (CLARK \& FUJIMOTO, 1991; GRIFFIN, 1997; LEE, LEE, \& SONDER, 2000; LOCH, STEIN, E TERWIESCH, 1996.). Um estudo de Stalk e Hout (1990) mostra que a velocidade de introdução de novos produtos está diretamente 
relacionada à posição de mercado, lucro e custos. Segundo Barnett e Clark (1998), os produtos têm uma vida útil limitada e precisam ser aperfeiçoados, desenvolvidos e inovados se a empresa deseja manter-se competitiva.

Segundo Filippini, Salmaso e Tessarolo (2004), o desenvolvimento de novos produtos de forma rápida transformou-se em uma prioridade em muitas organizações que se apressam para comercializar tecnologias emergentes e para satisfazer as necessidades dos clientes. Assim, está claro que um PDP é essencial para que as empresas possam competir com sucesso no mercado global. Existe um número crescente de empresas que estão investindo significativamente em iniciativas e recursos para desenvolver melhores práticas e para avaliar as práticas do PDP das empresas líderes do setor (LIM, SHARKEY e HEINRICHS, 2003).

O DP representa uma alternativa para que as pequenas e médias empresas aproveitem suas ideias inovadoras e estejam, geralmente, em uma posição principal para identificar as oportunidades do novo produto, devido às suas relações de trabalho próximas com clientes, fornecedores e concorrentes. O DP que envolve inovações radicais traz grandes oportunidades às empresas em termos de crescimento e da expansão em novos segmentos de mercado, além de possibilitar uma melhor posição competitiva (XIN, YENG E CHENG, 2008; DOOLEY E JOHNSON, 2001). O DP fornece uma plataforma para o sucesso financeiro em longo prazo, mas o PDP é um empreendimento complexo, arriscado e caro (MILLWARD et al., 2006; CHANDRA e NEELANKAVIL, 2008). As inovações radicais ajudam a manter o crescimento das vendas e o retorno das vendas (KOUFTEROS et al., 2005).

Muitos trabalhos já foram desenvolvidos no sentido de propor modelos de referência para o PDP, bem como adaptações desses modelos genéricos para determinados segmentos de mercado, os chamados modelos adaptados. Entretanto, esses diversos trabalhos estão distribuídos ao longo da base de conhecimento e ainda não foi identificado nenhum estudo que pudesse classificá-los, de modo a facilitar os projetos de pesquisa que visem propor novos modelos adaptados para segmentos de mercado ainda não explorados ou validar os modelos já existentes. 
Neste sentido, o objetivo geral deste presente trabalho é captar o estado da arte sobre o PDP e dos modelos de referência para o PDP. A partir de uma revisão de trabalhos publicados na literatura científica pretende-se identificar áreas nas quais podem ser desenvolvidos modelos de PDP específicos, de forma a beneficiar mais setores industriais. Assim, esse trabalho pode ser caracterizado como teóricoconceitual.

Para Godinho Filho e Fernandes (2004), a importância das classificações dos trabalhos dentro da atividade científica é clara, visto que o conhecimento científico se baseia na classificação. Portanto, a realização de uma classificação é ferramenta essencial para o conhecimento de determinada área. Neste artigo, a área definida é a de desenvolvimento de produtos, mais especificamente de modelos de referência para o desenvolvimento de produtos.

Portanto, a classificação dos modelos de referência já propostos para o PDP permite que outros pesquisadores possam identificar lacunas na literatura sobre modelos de desenvolvimento de produtos, favorecendo que novos modelos de referência sejam elaborados para setores industriais e arranjos produtivos locais (APL) ainda não estudados, contribuindo para o ensino e o aprendizado destes processos; contribua na divulgação de modelos de referência para o PDP já existentes e evidencie os ganhos da adoção de um processo de desenvolvimento de produtos mais formal e sistemático, integrado aos demais processos empresariais, com os participantes da cadeia de fornecimento e com os clientes finais.

\section{PROCESSO DE DESENVOLVIMENTO DE PRODUTOS}

Para Jun e Suh (2008), o PDP é complexo e não é organizado quando comparado com os processos de manufatura, porque possui características iterativas, evolucionárias, incertas, e cooperativas. Com a finalidade de controlar de forma eficaz o PDP é necessário a descrição das atividades, dos estágios e a lógica do processo. Isso exige uma estrutura de modelagem que possa capturar as características específicas 
de cada empresa. Entretanto, as metodologias de modelagem precedentes são limitadas na representação das características.

Para Cooper e Kleinschmidt (1995), o desempenho do PDP depende das atividades especificadas do processo, do programa da organização, da estratégia, da cultura e do clima organizacional, além do gerente de projetos.

Embora muitas empresas saibam da importância do PDP no desenvolvimento dos negócios a um longo prazo, por maior que seja o esforço da direção para melhoria do PDP, ainda assim a taxa de falhas dos novos produtos é elevada. Existem várias razões para estas elevadas taxas de falhas, sendo que uma das mais significativas é a baixa utilização de modelos, ferramentas e técnicas para auxiliar o PDP (NIJSSEN e FRAMBACH, 2000; GONZÁLEZ e PALÁCIOS, 2002; RUNDQUIST e CHIBBA 2004; YEH, PAI e YANG, 2008; CHANDRA e NEELANKAVIL, 2008).

Assim, Thia et al. (2005) afirmam que a utilização e a execução eficaz dessas ferramentas e técnicas do PDP são um elemento importante no gerenciamento integrado do PDP. As numerosas ferramentas e técnicas foram desenvolvidas por vários anos para fazer o PDP mais gerenciável. O uso e a aplicação de ferramentas da qualidade e as técnicas (incluindo modelos de desenvolvimento de produtos), quando utilizados dentro de uma metodologia eficaz de resolução de problemas, são essenciais para facilitar na melhoria de alguns processos. Para o processo de desenvolvimento isso não é nenhuma exceção (SPRING et al., 1998). Na pesquisa realizada por Chai e Xin (2006), os resultados revelaram que a aplicação das ferramentas de PDP é ainda pouco explorada na maioria das empresas em Singapura.

Em pesquisa realizada em Taiwan, Yeh, Pai e Yang (2008) afirmam que as ferramentas e as técnicas de NPD podem ser usadas por empresas para melhorar seu desempenho no desenvolvimento de novos produtos. Entretanto, muitas ferramentas e técnicas, potencialmente úteis, não são amplamente utilizadas pelas empresas. Parece que as razões para esta situação são: (i) que as empresas não compreendem claramente em que etapas as ferramentas e técnicas determinadas para o PDP podem ser usadas apropriada e eficazmente; (ii) que as empresas não verificaram a eficácia de 
várias ferramentas e de técnicas do PDP; e (iii) que os coordenadores do PDP não conhecem determinadas ferramentas e técnicas.

Dessa forma, os resultados dessas pesquisas supracitadas poderiam retratar uma situação parecida em diversas empresas no Brasil, especialmente as de micro, pequeno e médio porte. Isso ressalta a importância de se estudar os modelos propostos para o processo de desenvolvimento de produtos.

\section{MODELOS DE DESENVOLVIMENTO DE PRODUTOS}

A definição de um modelo de referência para o processo de desenvolvimento de produtos de empresas pode vir a contribuir para que as empresas do setor passem a executar um processo de desenvolvimento de produtos mais formal e sistemático, integrado aos demais processos empresariais, com os participantes da cadeia de fornecimento e com os clientes finais fornecer ainda, os meios para que as empresas inovem e desenvolvam novos produtos dentro de suas fábricas (FASS et al., 2009)

Para Smith e Marrow (1999), nos últimos anos, uma atenção maior tem sido dada à construção de modelos de processo de desenvolvimento de produtos. Compreender e modelar o processo é a primeira etapa importante na construção do modelo. O desenvolvimento de produtos é um processo complexo e os modelos serão úteis para auxiliar nas decisões gerenciais.

Segundo Vernadat (1996), modelos de referência são modelos parciais que podem ser usados como base para o desenvolvimento ou avaliação de modelos específicos. São chamados parciais por não atenderem ao processo existente em uma determinada realidade. Conforme Browning, Fricke e Negele (2006), um modelo é uma representação abstrata da realidade que é construída, verificada, analisada e manipulada para aumentar a compreensão dessa realidade. Os modelos podem residir na mente (modelos mentais) ou ser codificados.

Muitos autores buscam representar a realidade do desenvolvimento de produto por meio de modelos de referência. Os modelos auxiliam na concepção de uma visão 
única do PDP, descrevendo-o e servindo de referência para que empresas e seus profissionais possam desenvolver produtos segundo um padrão estabelecido. Os modelos referenciais podem ser elaborados para serem aplicados para atender um tipo de organização, um setor industrial, uma arranjo produtivo local, ou seja, pode ser aplicado a qualquer tipo de PDP (MENDES, 2008). Isso é confirmado por Schmidt, Sarangee, Montoya (2009), que afirmam que diferentes organizações têm números diferentes de estágios, de atividades e de pontos da tomada de decisão no seu PDP.

Além disso, Bujis (2003) afirma que dependendo dos objetivos e metas da empresa deve-se escolher um modelo específico, uma versão específica do modelo ou um nível de detalhamento específico.Para Engwall, Kling e Werr (2005) mesmo em uma simples forma, os modelos de desenvolvimento de produtos consistem em um esboço das principais atividades durante o processo do desenvolvimento de produtos, apresentando as melhores práticas desse processo.

Como os modelos são interpretados pelos seus usuários e aplicados nas empresas, ocorrem diversas maneiras de usar um mesmo modelo. Diferentes usuários enfatizam diferentes características do modelo e usam estas características das mais variadas formas. Dessa forma, a prática do desenvolvimento de produtos pode ser melhorada pela execução de novos modelos. Isso é foi mostrado em pesquisa realizada por Zancul, Marx e Metzker (2006) em duas empresas automobilísticas.

O modelo pode contribuir para facilitar a compreensão e a comunicação entre os coordenadores do projeto do produto, para auxiliar nas decisões quando necessário e para auxiliar as funções da gestão do projeto, tais como o planejamento e organização. No PDP é importante usar a modelagem de ferramentas ou de diagramas para descrever as características do processo para facilitar o gerenciamento e a análise (JUN e SUH, 2008).

Sendo assim, pode-se definir modelo de referência como sendo a união das melhores práticas relacionadas a um determinado processo de desenvolvimento, sendo estas representadas de uma forma clara a qualquer usuário deste processo. 


\section{CLASSIFICAÇÃO DOS MODELOS}

Este trabalho é do tipo teórico-conceitual e realiza uma revisão bibliográfica sobre estudos que falam sobre modelos de PDP específicos. Para isso, é utilizada a estrutura metodológica adaptada proposta por Godinho Filho \& Fernandes (2003). Essa estrutura é mostrada na Figura 1, na qual pode-se notar que a realização de uma revisão sobre modelos de PDP deve ser o passo inicial para este tipo de trabalho. As publicações de interesse foram localizadas por meio de consultas nas bases de dados dos periódicos da CAPES (Coordenação de Aperfeiçoamento de Pessoal de Nível Superior). A utilização dessa base de dados é justificada pela sua grande abrangência e facilidade de acesso para a maioria dos pesquisadores no Brasil. Inicialmente, é importante destacar que para identificar, localizar e adquirir as publicações de interesse, foram consultadas as seguintes bases de dados, disponíveis no portal periódicos da CAPES: Emerald, ProQuest, SciELO, Science Direct, Springer e Wilson. Ainda no mesmo portal, foi realizada uma busca no banco de teses e dissertações. Analogamente, foi realizada uma busca no Google acadêmico com a finalidade de encontrar os artigos referentes a congressos nacionais e livros correlatos.

Para realizar os fichamentos dos trabalhos, utilizou-se uma adaptação das tabelas usadas nos levantamentos de Carnevalli e Miguel (2007), Godinho Filho e Fernandes (2004), Godinho Filho e Fernandes (2003), Miguel (2007) e Godinho Filho, Fernandes e Lima (2009). Assim, a classificação dos artigos foi codificada conforme apresentado no quadro 1. No quadro 2 é apresentado um detalhamento dos 35 modelos estudados, codificados conforme o quadro 1 . 


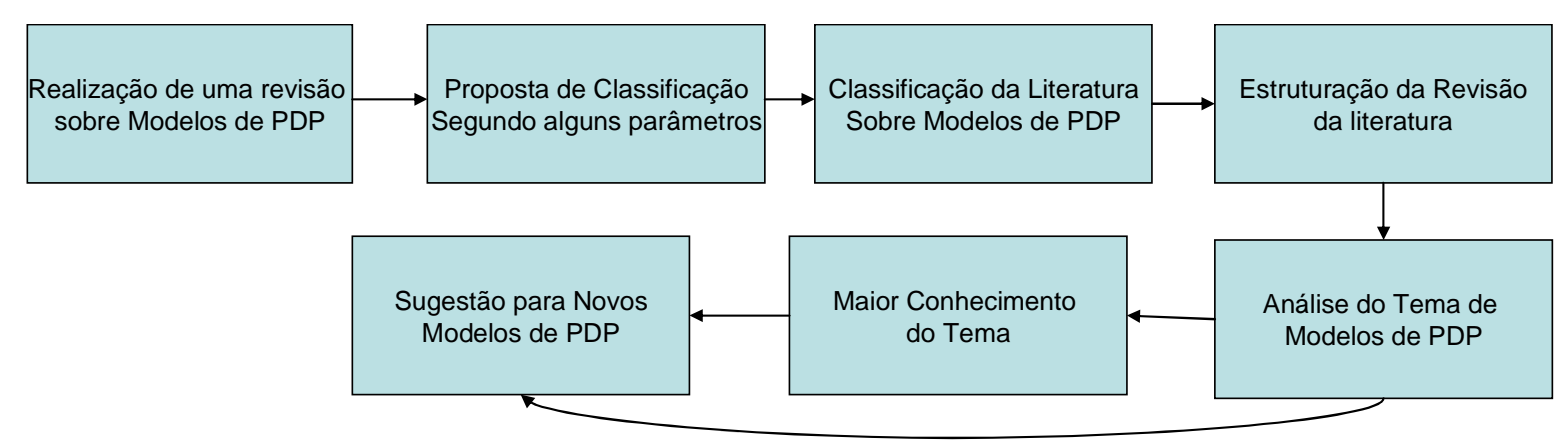

Figura 1 - Estrutura do trabalho

Fonte: adaptado de Godinho Filho e Fernandes (2003)

A tarefa de classificação dos modelos partiu das classificações descritas nos próprios trabalhos e da análise e reflexão dos autores do presente trabalho. Os trabalhos foram classificados com relação ao tipo de estudo (modelagem, teóricoconceitual, revisão da literatura, simulação, survey, estudo de caso, pesquisa-ação e experimentação). Com relação à abordagem, os trabalhos também foram classificados em quantitativo e qualitativo. Foi verificada a abrangência do modelo (regional, nacional ou internacional); qual a unidade de análise (pessoas, grupos, unidade organizacional ou empresas); a forma predominante na coleta de dados (questionário, entrevistas; análise documental, dados públicos, dados da imprensa ou bibliográfico), período analisado (retrospectiva, atual ou longitudinal). Foram verificados ainda os benefícios tangíveis e intangíveis, filiação dos autores (centro de pesquisa, universidade, ou empresa) e o tipo de documento (artigo internacional, artigo nacional, artigo de congresso, dissertação, tese ou livro).

Inicialmente buscou-se nos periódicos pelos seguintes termos: "new product development process" e "models" em todo o texto. Posteriormente, buscou-se pelos termos "product development process" e "models", "product development process modelling" e finalizou-se com "models of product development". Os mesmos termos, em português, foram utilizados para a busca na base de teses e dissertações. O período pesquisado foi a partir de 1995. 


\begin{tabular}{|c|c|c|c|}
\hline Identificação & Codificação & Identificação & Codificação \\
\hline T1 & Tipo de estudo & T8 & Documentação \\
\hline A & Modelagem & 1 & Questionário \\
\hline $\mathrm{B}$ & Teórico-conceitual & 2 & Entrevistas \\
\hline C & Revisão de literatura & 3 & Análise documental \\
\hline $\mathrm{D}$ & Simulação & 4 & Dados públicos \\
\hline$E$ & Survey & 5 & Dados da imprensa \\
\hline $\mathrm{F}$ & Estudo de caso & 6 & Bibliográfico \\
\hline $\mathrm{G}$ & Pesquisa-ação & T9 & Benefícios tangíveis \\
\hline $\mathrm{H}$ & Experimental & B1 & Melhorar a confiabilidade \\
\hline T2 & Filiação & $\mathrm{B} 2$ & Reduzir alterações no projeto \\
\hline UN & Universidade & B3 & Reduzir o tempo \\
\hline $\mathrm{CP}$ & Centro de pesquisa & B4 & Reduzir os custos \\
\hline EP & Empresa & B5 & $\begin{array}{l}\text { Outros benefícios tangíveis } \\
\text { referentes à melhoria do projeto }\end{array}$ \\
\hline T3 & Apoio financeiro & B6 & Benefícios tangíveis fora do projeto \\
\hline AF & Apoio financeiro & B15 & $\begin{array}{l}\text { Identificar/ressaltar particularidades } \\
\text { do modelo de DP }\end{array}$ \\
\hline T4 & Período de análise & B16 & $\begin{array}{l}\text { Estender a aplicabilidade do } \\
\text { modelo de DP }\end{array}$ \\
\hline LO & Longitudinal & T10 & Benefícios intangíveis \\
\hline RET & Retrospectivo & B7 & Método flexível \\
\hline AT & Atual & B8 & Melhoria da comunicação \\
\hline T5 & Abordagem & B9 & $\begin{array}{l}\text { Ajudar na análise dos dados e na } \\
\text { tomada de decisão racional }\end{array}$ \\
\hline a & Quantitativo & B10 & Trabalho em equipe \\
\hline $\mathrm{b}$ & Qualitativo & B11 & $\begin{array}{l}\text { Melhorar o knowhow e a sua } \\
\text { conservação }\end{array}$ \\
\hline T6 & $\begin{array}{l}\text { Abrangência } \\
\text { geográfica }\end{array}$ & B12 & $\begin{array}{l}\text { Integração de ferramentas e } \\
\text { métodos }\end{array}$ \\
\hline $\mathrm{RE}$ & Regional & B13 & $\begin{array}{l}\text { Outros benefícios intangíveis } \\
\text { referentes à melhoria do projeto }\end{array}$ \\
\hline NA & Nacional & B14 & $\begin{array}{l}\text { Benefícios intangíveis fora do } \\
\text { projeto }\end{array}$ \\
\hline IN & Internacional & T11 & Caracterização dos modelos \\
\hline T7 & Unidade de análise & DP & Densenvolvimento de produtos \\
\hline PE & Pessoas & GDP & Gestão do DP \\
\hline GR & Grupos & Misto & $\mathrm{DP}+\mathrm{GDP}$ \\
\hline $\mathrm{O}$ & $\begin{array}{l}\text { Unidades } \\
\text { organizacionais }\end{array}$ & T12 & Tipo de documento \\
\hline \multirow[t]{6}{*}{ EMP } & Empresas & DIS & Dissertação \\
\hline & & TES & Tese \\
\hline & & ARN & Artigo de revista nacional \\
\hline & & ARI & Artigo de revista internacional \\
\hline & & $\mathrm{ACO}$ & Artigo de congresso \\
\hline & & LIV & Livro \\
\hline
\end{tabular}

Quadro 1 - Codificação para classificação dos trabalhos

Revista Produção Online, v.10, n.4, p. 886-911, dez., 2010 


\begin{tabular}{|c|c|c|c|c|c|c|c|c|c|c|c|c|c|}
\hline & \multirow{2}{*}{\multicolumn{12}{|c|}{ Classificação }} \\
\hline & \multirow[b]{2}{*}{ Área de aplicação } & & & & & & & & & & & & \\
\hline Autor(es) & & T1 & T2 & T3 & T 4 & T 5 & T 6 & $\mathbf{T} 7$ & T 8 & T 9 & $\mathbf{T} 10$ & T 11 & $\mathbf{T} 12$ \\
\hline Fuller (1994) & Produtos alimentícios & $\mathrm{B}$ & EP & & RET & $b$ & IN & $\mathrm{O}$ & 6 & B6 & B11 & $\mathrm{DP}$ & LIV \\
\hline Rudolph (1995) & Produtos alimentícios & $\mathrm{B}$ & UN & & AT & $b$ & IN & EMP & 6 & B15 & B9 & Misto & ARI \\
\hline Hunt e Jones (1998) & $\begin{array}{l}\text { Produtos eletrônicos e de } \\
\text { tecnologia de informação }\end{array}$ & $\mathrm{B}$ & UN & & AT & $b$ & IN & EMP & 6 & B5 & B9 & Misto & ARI \\
\hline Ledwith (2000) & $\begin{array}{l}\text { Produtos para pequenas } \\
\text { empresas eletrônicas }\end{array}$ & $A$ & UN & & AT & $b$ & RE & EMP & 1 & B6 & B14 & DP & ARI \\
\hline $\begin{array}{l}\text { Bumgardner, Bush e } \\
\text { West (2001) }\end{array}$ & Produtos moveleiros & $E$ & UN & $\mathrm{AF}$ & AT & $b$ & RE & EMP & 1 & B5 & B11 & $\mathrm{DP}$ & ARI \\
\hline Nwabueze e Law (2001) & Produtos para cervejaria & $\mathrm{F}$ & UN & & AT & $\mathrm{b}$ & RE & EMP & 2 & B15 & B11 & Misto & ARI \\
\hline Fabrício (2002) & $\begin{array}{l}\text { Novos empreendimentos na } \\
\text { construção civil }\end{array}$ & $\mathrm{F}$ & UN & $\mathrm{AF}$ & RET & $b$ & NA & EMP & 2 & B16 & B12 & GDP & TES \\
\hline $\begin{array}{l}\text { Formoso, Tzortzopoulos } \\
\text { e Liedtke (2002) }\end{array}$ & $\begin{array}{l}\text { Construção para casas e } \\
\text { comércio }\end{array}$ & $\mathrm{G}$ & UN & $\mathrm{AF}$ & AT & $b$ & RE & EMP & 2 & B1 & B11 & DP & ARI \\
\hline Romano (2003) & Máquinas agrícolas & $\mathrm{C}$ & UN & & RET & $\mathrm{b}$ & NA & 0 & 6 & B15 & B11 & DP & TES \\
\hline $\begin{array}{lll}\text { Toni } \\
(2003)\end{array}$ & $\begin{array}{l}\text { Óculos para pequenas e } \\
\text { médias empresas }\end{array}$ & C & UN & & AT & & NA & PE & 3 & B16 & B12 & $\mathrm{DP}$ & DIS \\
\hline Montemezzo (2003) & Produtos da moda & $\mathrm{F}$ & UN & AF & AT & $b$ & NA & EMP & 2 & B16 & B12 & DP & TES \\
\hline Bujis (2003) & Produtos inovativos & $\mathrm{F}$ & UN & & AT & $\mathrm{b}$ & $\mathrm{RE}$ & EMP & 2 & B15 & $\mathrm{B} 12$ & Misto & $\mathrm{ARI}$ \\
\hline $\begin{array}{l}\text { Gómez, Vidal e } \\
\text { Alcamí (2004) }\end{array}$ & $\begin{array}{l}\text { Produtos para empresas } \\
\text { espanholas do setor } \\
\text { cerâmico }\end{array}$ & $\mathrm{F}$ & UN & & AT & $b$ & $R E$ & EMP & 2 & B16 & B12 & GDP & $A R I$ \\
\hline Thier (2005) & $\begin{array}{l}\text { Produtos para indústria de } \\
\text { cerâmica vermelha }\end{array}$ & $\mathrm{G}$ & UN & AF & AT & $b$ & NA & EMP & 1 & B16 & B12 & DP & DIS \\
\hline
\end{tabular}

Quadro 2 - Apresentação dos artigos codificados por anos de publicação (continua) 


\begin{tabular}{|c|c|c|c|c|c|c|c|c|c|c|c|c|c|}
\hline & \multirow{2}{*}{\multicolumn{12}{|c|}{ Classificação }} \\
\hline & & & & & & & & & & & & & \\
\hline Autor(es) & Área de aplicação & T1 & T2 & T3 & T 4 & T 5 & T 6 & T 7 & T 8 & T 9 & $\mathrm{~T} 10$ & $\mathrm{~T} 11$ & $\mathrm{~T} 12$ \\
\hline $\begin{array}{l}\text { Zancul e Rozenfeld } \\
\text { (2005) }\end{array}$ & Produtos populares & $\mathrm{F}$ & UN & & AT & $\mathrm{b}$ & $\mathrm{RE}$ & GR & 2 & B16 & B12 & $\mathrm{DP}$ & $\mathrm{ACO}$ \\
\hline Paula e Cheng (2005) & $\begin{array}{l}\text { Produtos para empresas de } \\
\text { base tecnológica }\end{array}$ & $\mathrm{F}$ & UN & $\mathrm{AF}$ & AT & $b$ & $\mathrm{RE}$ & EMP & 2 & B5 & $\mathrm{B} 12$ & GDP & TES \\
\hline Trim e Pam (2005) & Produtos farmacêuticos & $\mathrm{G}$ & UN & & LO & $b$ & $\mathrm{RE}$ & $\mathrm{O}$ & 1 & B5 & B12 & Misto & TES \\
\hline Delgado Neto (2005) & Jogos e brinquedos infantis & C & UN & & AT & $\mathrm{b}$ & $\mathrm{RE}$ & & 6 & & B12 & GDP & $\mathrm{ACO}$ \\
\hline Mello (2005) & Serviço & $\mathrm{F}$ & UN & $\mathrm{AF}$ & AT & $b$ & $\mathrm{RE}$ & EMP & 2 & B5 & B12 & GDP & TES \\
\hline Barbalho (2006) & Produtos mecatrônicos & G & UN & & LO & $b$ & $\mathrm{RE}$ & $\mathrm{O}$ & 1 & B5 & B12 & Misto & TES \\
\hline Alvarenga (2006) & Produtos inclusivos & $\mathrm{F}$ & UN & AF & AT & $\mathrm{b}$ & $\mathrm{RE}$ & $\mathrm{PE}$ & 1 & B4 & $\mathrm{B} 14$ & Misto & TES \\
\hline Facó (2006) & Novos produtos em bancos & $\mathrm{F}$ & UN & AF & AT & $b$ & NA & $\mathrm{O}$ & 2 & B6 & B14 & GDP & DIS \\
\hline Rozenfeld et al. (2006) & $\begin{array}{l}\text { Autopeças e empresas da } \\
\text { linha branca }\end{array}$ & C & UN & & AT & $b$ & NA & & 6 & & $\mathrm{~B} 12$ & GDP & LIV \\
\hline $\begin{array}{l}\text { MacGregor, Arana, } \\
\text { Parra e Lorenzo (2006) }\end{array}$ & $\begin{array}{l}\text { Produtos para centros de } \\
\text { pesquisa tecnológicas }\end{array}$ & $\mathrm{F}$ & $\mathrm{CP}$ & & LO & $b$ & $\mathrm{RE}$ & EMP & 6 & B3 & B12 & GDP & ARI \\
\hline Prasnikar e Skerlj (2006) & $\begin{array}{l}\text { Produtos para empresas } \\
\text { farmacêuticas }\end{array}$ & $\mathrm{F}$ & UN & & RET & $\mathrm{b}$ & IN & EMP & 1 & B6 & B9 & GDP & ARI \\
\hline Paula e Ribeiro (2007) & Produtos farmacêuticos & $\mathrm{F}$ & UN & AF & AT & $b$ & NA & EMP & 2 & B6 & B12 & GDP & ARI \\
\hline $\begin{array}{l}\text { Ottenbacher e } \\
\text { Harrington (2007) }\end{array}$ & Produtos alimentícios & $\mathrm{F}$ & UN & & AT & $\mathrm{b}$ & NA & EMP & 2 & B6 & B14 & GDP & ARI \\
\hline Yeh, Pai e Yang (2008) & $\begin{array}{l}\text { Produtos para empresas de } \\
\text { alta tecnologia }\end{array}$ & $\mathrm{F}$ & UN & & AT & $b$ & NA & $\mathrm{O}$ & 1 & B5 & $\mathrm{B} 12$ & GDP & ARI \\
\hline Mendes (2008) & $\begin{array}{l}\text { Produtos para EBT, MPE de } \\
\text { automação de controle de } \\
\text { processos e de equipamentos } \\
\text { médico-hospitalares }\end{array}$ & $\mathrm{F}$ & UN & & AT & $b$ & $\mathrm{RE}$ & $\mathrm{O}$ & 1 & B6 & $\mathrm{B} 12$ & GDP & TES \\
\hline
\end{tabular}

Quadro 2 - Apresentação dos artigos codificados por anos de publicação (continua)

Revista Produção Online, v.10, n.4, p. 886-911, dez., 2010 


\section{Classificação}

\begin{tabular}{|l|l|l|l|l|l|l|l|l|l|l|l|l|l|}
\hline \multicolumn{1}{|c|}{ Autor(es) } & \multicolumn{1}{|c|}{ Área de aplicação } & T1 & T2 & T3 & T 4 & T 5 & T 6 & T 7 & T 8 & T 9 & T 10 & T 11 & T 12 \\
\hline Maluf Filho (2008) & Pneus automobilísticos & A & UN & & AT & b & NA & & & B1 & B12 & GDP & Tes \\
\hline Colenci Neto (2008) & Software & G & UN & & AT & b & RE & O & 1 & B5 & B12 & GDP & Tes \\
\hline Miller e Clarke (2008) & Aeronaves & F & UN & & AT & b & RE & O & & B1 & B7 & GDP & ARI \\
\hline $\begin{array}{l}\text { Marion e Simpson } \\
\text { (2009) }\end{array}$ & $\begin{array}{l}\text { Produtos para firmas } \\
\text { nascentes }\end{array}$ & G & UN & & AT & b & RE & O & & B5 & B7 & GDP & ARI \\
\hline Neto e Nobre (2009) & Produtos imobiliários & G & UN & AT & b & RE & O & 2 & B5 & B12 & GDP & ARN \\
\hline Suarez et. Al (2009) & Produtos decorativos & G & UN & AT & b & RE & O & 2 & B5 & B14 & Misto & ARN \\
\hline
\end{tabular}

Quadro 2 - Apresentação dos artigos codificados por anos de publicação (conclusão 


\section{RESULTADO DA CLASSIFICAÇÃO DOS MODELOS}

Com relação ao tipo de estudo, foi possível perceber que muitos autores não definem adequadamente o método empregado nas suas pesquisas. A figura 2 classifica mais detalhadamente as pesquisas realizadas com relação ao tipo de estudo, sendo que os valores apresentados nas colunas se referem às porcentagens em relação ao total de pesquisas pelo tipo de estudos. Verifica-se na figura 2 que a pesquisa classificada como estudo de caso foi a mais realizada nos trabalhos estudados e, em segundo lugar, se destacam as pesquisas do tipo pesquisa-ação e revisão da literatura. Ainda sobre a figura 2, não foram encontradas pesquisas sobre o modelos para o PDP classificadas como simulação ou experimental.

A figura 3 apresenta nas colunas as porcentagens em relação ao total dos trabalhos analisados pelo tipo de documentação utilizada. Percebe-se que $75 \%$ da documentação utilizada foram as entrevistas e questionários, mas não foram utilizados dados públicos e dados da imprensa para elaboração dos modelos. Na pesquisa realizada na literatura identificada evidenciou-se que 100\% dos trabalhos foram classificados como qualitativos.

A literatura pesquisada, como por exemplo, Back (1983), Vincent (1989), Rosenthal (1992), Wheelwright \& Clark (1992), Cooper \& Edgett (1999), Pahl et al. (2005) e Rozenfeld et al. (2006), mostra que cada autor interpreta o processo de desenvolvimento de produtos por uma diferente ótica. A maioria destes modelos são apresentados em etapas ou em estágios e, devido a essa generalização, podem variar substancialmente de indústrias para indústrias. Muitos modelos do processo do desenvolvimento de produtos são similares, entretanto, diferenciam-se na quantidade de etapas ou estágios. 


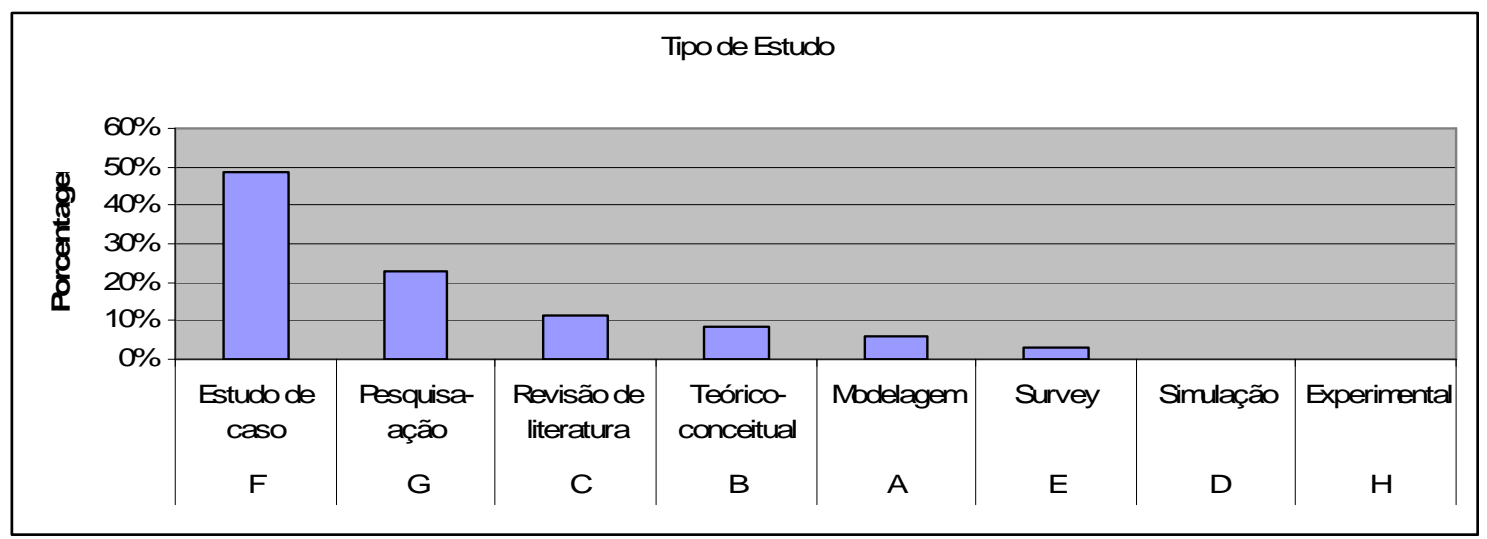

Figura 2 - Tipo de Estudo

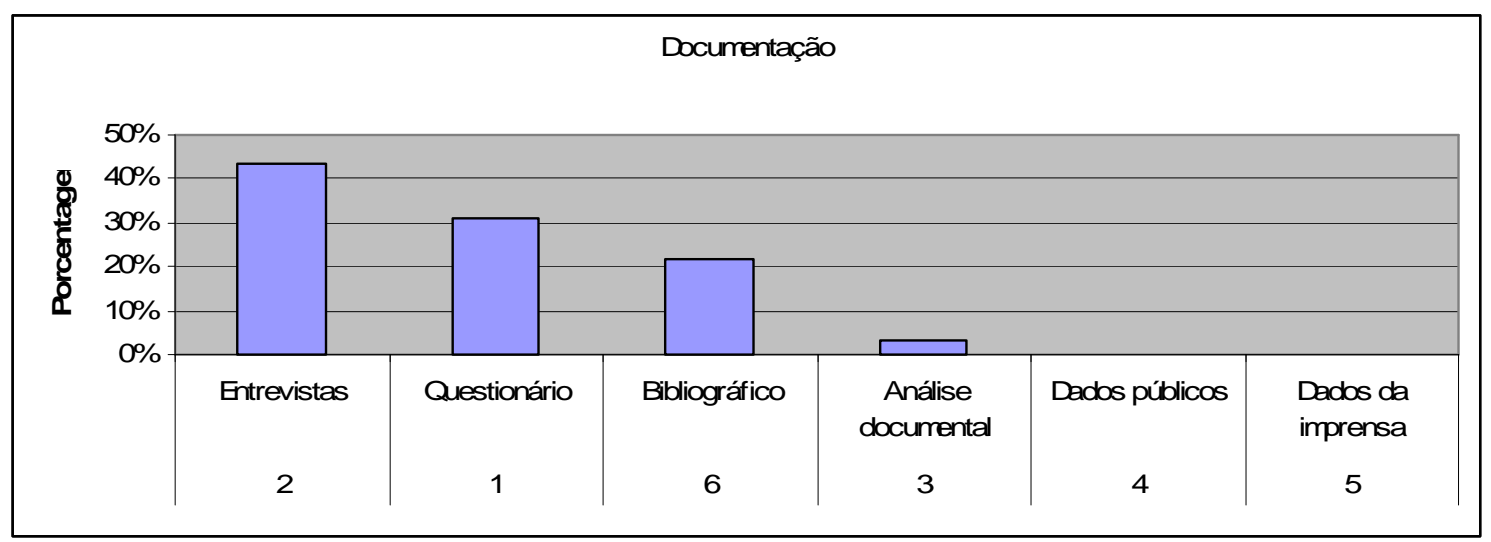

Figura 3 - Documentação

Vale ressaltar que os modelos possuem diferentes níveis de detalhamento, de acordo com a necessidade da empresa, setor ou ramo em que ela atua. Porém, muitas fases são encontradas em vários modelos, mas com denominações diferentes. Isso pode ser justificado pela figura 4 que ilustra o nível de generalização do PDP, sendo que cada empresa pode definir seu modelo, assim como as etapas, fases e atividades que melhor se adéquem às suas práticas. Isso foi evidenciado no levantamento que apurou que em $54 \%$ dos casos pesquisados a abordagem geográfica foi classificada como sendo regional, como mostra a figura 5 e em $89 \%$ dos casos realizados nas empresas. Analisando a figura 6, percebe-se que $75 \%$ dos trabalhos são artigos internacionais ou teses, mostrando a importância e a relevância do tema. Mesmo assim, o número de livros para PDP específicos ainda é baixo. 
Verificando setores considerados estratégicos por vários países, como o militar, biotecnológico e o naval, não foram encontrados relatos de modelos de gestão do PDP. Para os países como o Brasil, que estão investindo em processos de extração do petróleo em águas profundas, seria interessante a preocupação com o desenvolvimento de um modelo para gerenciar o PDP desse setor. Além disso, devido ao crescimento do número de APL's em todo o país, é de grande interesse o desenvolvimento de produtos de modelos de PDP específicos para cada APL ou ainda para Empresas de Base Tecnológica (EBT) de setores específicos.

Com a revisão da literatura em modelos de desenvolvimento de produtos, percebeu-se que a seleção para a adequação de um modelo específico para uma empresa ou ramo de indústria é feito apenas sob a ótica do pesquisador, utilizando como justificativas convencionais o fato do modelo ser mais o citado na literatura, ser o mais recente ou, até mesmo, a proximidade entre os setores industriais. Por este aspecto, o levantamento mostrou que em $94 \%$ dos trabalhos os pesquisadores estavam ligados a uma universidade e todos utilizaram uma abordagem qualitativa para a proposição dos modelos.

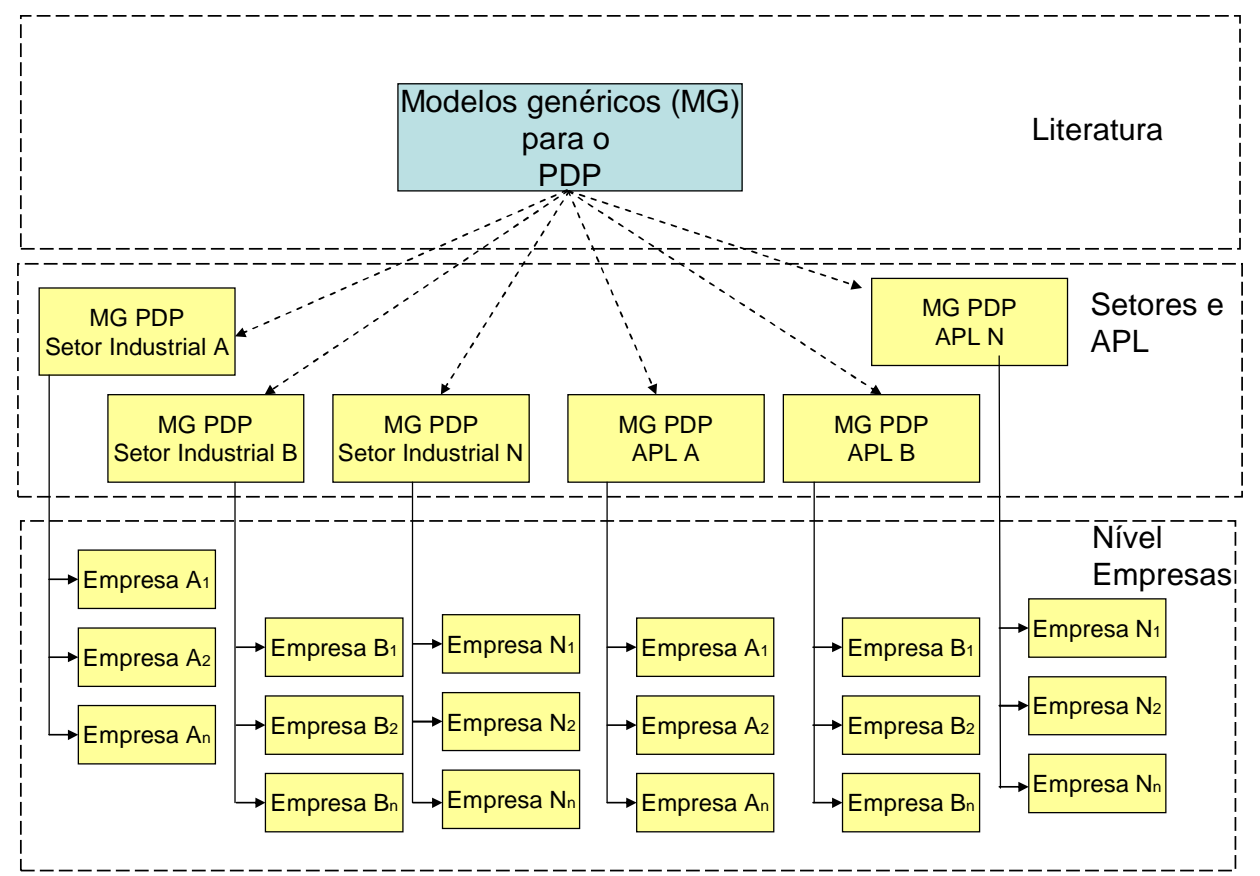

Figura 4 - Níveis de generalização do PDP

Revista Produção Online, v.10, n.4, p. 886-911, dez., 2010 


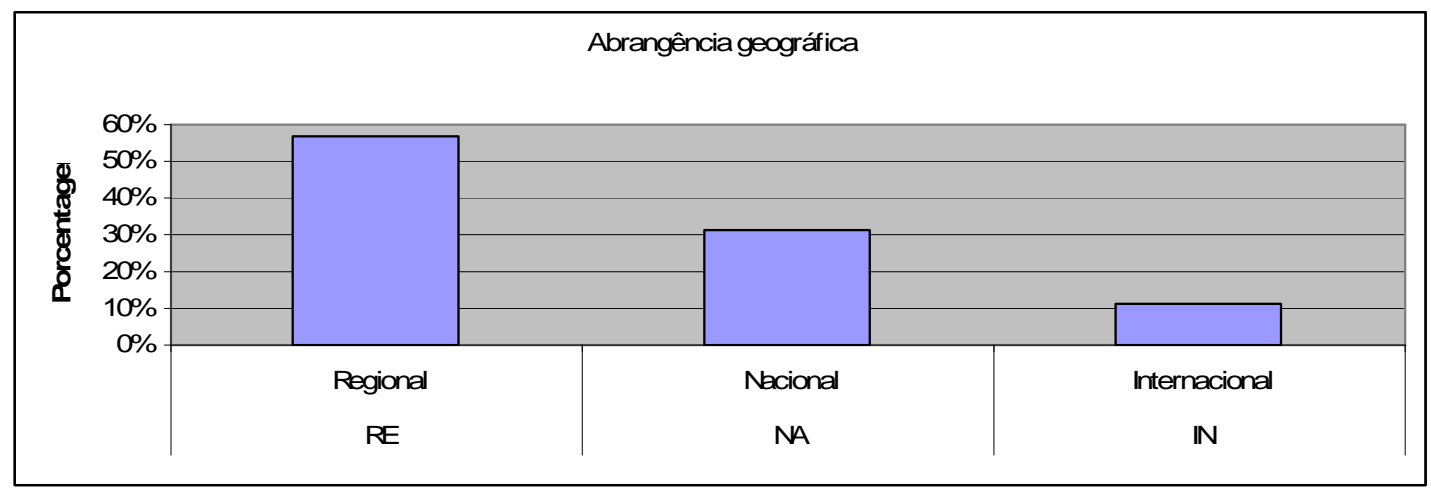

Figura 5 - Abrangência Geográfica dos modelos de PDP

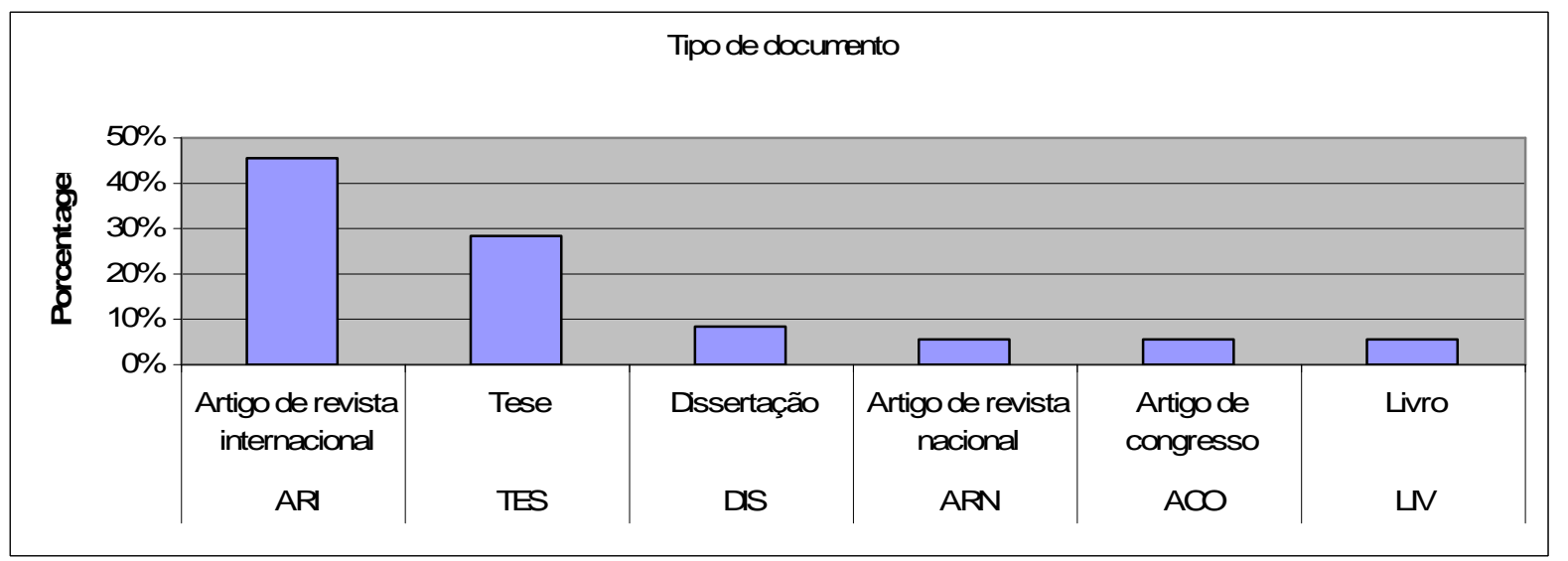

Figura 6 - Tipos de documentos

Os pesquisadores poderiam selecionar os modelos conforme Fass et al. (2009), que na sua pesquisa selecionaram o modelo genérico que melhor se adequou ao processo de desenvolvimento de produtos para empresas de base tecnológica do APL de Santa Rita do Sapucaí utilizando um método de auxílio a decisão, o AHP. Para Wang, Chu e Wu (2007 o AHP é o mais popular método de decisão com múltiplos critérios que permite a medição da coerência dos julgamentos das decisões. Essa ferramenta contribui para a escolha do modelo mais adequado, propiciando uma escolha menos dependente dos conceitos do pesquisador. 


\section{CONCLUSÃO}

Com a conclusão deste trabalho percebeu-se que, até o momento, tem havido um maior enfoque das pesquisas acadêmicas em ressaltar o que é comum aos vários modelos de desenvolvimento de produtos, mas pouco enfoque vem sendo dado aos aspectos que os tornam diferentes e mais adequados a um ou outro tipo de negócio. Diante deste cenário, identifica-se uma tendência em realizar pesquisas focando modelos de desenvolvimento de produtos para determinados setores industriais.

O objetivo geral desse trabalho foi atingido, pois foi realizado um levantamento do estado da arte sobre os modelos do PDP e identificados os setores em que não foram encontrados modelos adaptados na literatura pesquisada. Assim, pode-se concluir que existe uma tendência de desenvolver modelos mais específicos, ou seja, para arranjos produtivos locais. Foram identificadas também lacunas em setores específicos da economia como o setor naval, biotecnológico ou militar, e até mesmo lacunas entre os modelos como, por exemplo, para EBT's de pequeno porte de um setor específico.

Outro fator importante é que os modelos de processo de desenvolvimento de produtos se confundem com os projetos, pois a maioria dos modelos são representados como se em escala temporal tivesse um início e um fim bem definidos, porém o processo é repetitivo e cíclico sendo necessário voltar a algumas fases ou etapas. Isso possivelmente ocorre devido ao fato de, didaticamente, ser mais fácil representar o PDP com um início e um fim definidos.

Como sugestão para trabalhos futuros recomenda-se a elaboração de modelos de desenvolvimento de produtos para as lacunas encontradas na literatura e o desenvolvimento de trabalhos que descrevam a aplicação desses modelos nas empresas (boa parte dos trabalhos são de cunho acadêmico), visando sua validação interna e, principalmente, externa.

Finalmente, consideram-se como as principais contribuições deste trabalho: maior aprofundamento sobre o tema pesquisado, propiciando oportunidades para que 
outros pesquisadores desenvolvam estudos em modelos de desenvolvimento de produtos; identificar lacunas para o desenvolvimento de modelos específicos em diversos segmentos de mercado; e a sugestão de oportunidades para futuras pesquisas dentro do tema.

\section{AGRADECIMENTOS}

A CAPES (Programa Pró-Engenharias - processo PE024/2008), a FAPEMIG (011/07 - TEC APQ 64126.01/07, Programa Jovens Pesquisadores - EDT-538/07, Programa Pesquisador Mineiro - TEC-PPM00043-08) e a UNIFAL pela liberação para realização da pesquisa.

\section{REFERÊNCIAS}

ALMEIDA, L.F.; MIGUEL, P. A. C. The first stage of a proposal of a theoretical model for managing a new product development process. Product: Management \& Development, v. 5, n.1, p. 57-64, 2007.

ALVARENGA, F. B. Uma abordagem metodológica para o projeto de produtos inclusivos. Universidade Estadual de Campinas, Faculdade de Engenharia Mecânica, tese de doutorado, Campinas/SP, 2006.

BACK, N. Metodologia de projeto de produtos industriais. Rio de Janeiro: Guanabara Dois: 1983.

BARBALHO, S. C. M. Modelo de referência para o desenvolvimento de produtos mecatrônicos: proposta e aplicações. Tese (Doutorado) - Escola de Engenharia de São Carlos, 2006.

BARNETT, B. D.; CLARK, K. B. Problem solving in product development: a model for the advanced materials industries. International Journal of Technology Management, v. 15, n. 8, p. $805-820,1998$.

BLACKBURN, J. D. Time-based competition: the next battleground in American manufacturing. Homewood, IL: Ed. Business One Irwin, 1991.

BROWNING, T. R.; FRICKE, E.; NEGELE, H.. Key Concepts in Modeling Product Development Processes. Systems Engineering, v. 9, n. 2, p. 104-128, 2006.

BUJIS, J.. Modelling Product Innovation Processes, from Linear Logic to Circular Chaos. Creativity and innovation management, v. 12, n. 2, 2003. 
BUMGARDNER, M. S.; BUSH, R. J.; WEST, C. D. Product development in large furniture companies: a descriptive model with implications for Character-marked products. Wood and Fiber Science, v. 33, n. 2, p. 302-313, 2001.

CARNEVALLI, J. A.; MIGUEL, P. A. C. Revisão, análise e classificação da literatura sobre o QFD - tipos de pesquisa, dificuldades de uso e benefícios do método. Gestão e Produção, v.14, n. 3, p.557-579, 2007.

CHAI, KAH-HIN; XIN, Y. The Application of New Product Development Tools in Industry: The Case of Singapore. IEEE transactions on engineering management, v. 53, n. 4, 2006.

CHANDRA, M.; NEELANKAVIL, J. Product development and innovation for developing countries: Potential and challenges. Journal of Management Development, v. 27, n. 10, p.1017-1025, 2008.

CLARK, K. B.; FUJIMOTO, T. Product development performance: strategy, organization and management in the word auto industry. Boston-Massachusetts: Harvard Business School Press, 1991.

COLENCI NETO, A. Proposta de um modelo de referência para desenvolvimento de software com foco na certificação do MPS.Br. Universidade de São Paulo, Escola de Engenharia de São Carlos - Departamento de Engenharia de Produção, tese de doutorado, São Carlos/SP, 2008.

COOPER, R. G.; EDGETT, S. J. Product development for de service sector: lessons from market leaders. New York: Basic Books, 1999.

COOPER, R. G.; KLEINSCHMIDT, E. J. Benchmarking the firm's critical sucess factors in New Product Development. Journal of Product Innovation Management, v. 12, p.374-391, 1995.

DELGADO NETO, G. G. Uma contribuição à metodologia de projeto para o desenvolvimento de jogos e brinquedos infantis. Universidade Estadual de Campinas, Faculdade de Engenharia Mecânica, 2005.

DOOLEY.K.; JOHNSON, D. Changing the new product development process: Reengineering or continuous quality improvement? Measuring Business Excellence, v. 5, n. 4, p.32-38, 2001.

ENGWALL, M.; KLING, R.; WERR, A. Models in action: how management models are interpreted in new product development. R\&D Management, v. 35, n. 4, p.427-439, 2005. 
FABRíCIO, M. M. Projeto simultâneo na construção de edifícios. Tese(Doutorado) Escola Politécnica, Universidade de São Paulo, São Paulo/SP, 2002.

FACÓ, J. F. B. O processo de desenvolvimento de novos produtos em ambientes de informação intensiva: uma análise exploratória aplicada ao setor de bancos de varejo do Brasil. Dissertação (Mestrado em Administração de Empresas) - Escola de Administração de Empresas de São Paulo da Fundação Getúlio Vargas, São Paulo/SP, 2006.

FASS, F. D. M.; SALGADO, E. G.; SALOMON, V. A. P.; MELLO, C. H. P.; SILVA, C. E. $\mathrm{S}$. Seleção de um modelo de processo de desenvolvimento de produto para indústria de base tecnológica do ramo eletroeletrônico. ENCONTRO NACIONAL DE ENGENHARIA DE PRODUÇÃO. 29. 2009. Anais ENEGEP. Salvador, 2009.

FILIPPINI, R.; SALMASO, L.; TESSAROLO, P. Product Development Time Performance: Investigating the Effect of Interactions between Drivers. Journal of Product Innovation Management, v. 21, n. 3, p.199-214, 2004.

FORMOSO, C. T.; TZORTZOPOULOS, P.; LIEDTKE, R. A model for managing the product development process in house building. Engineering, Construction and Architectural Management, v. 9, n. 5/6, p.419-432, 2002.

FULLER, G. W. New food product development: from concepts to marketplace.USA: CRC Press, 1994.

GODINHO FILHO, M.; FERNANDES, F. C. F. Manufatura enxuta: uma revisão que classifica e analisa os trabalhos apontando perspectivas de pesquisas futures. Gestão e Produção, v. 11, n. 01, p.1-19, 2004.

GODINHO FILHO, M.; FERNANDES, F. C. F. Um sistema para classificar e codificar os trabalhos que relacionam o controle da produção e o controle da qualidade. Gestão e Produção, v. 10, n. 1, p.89-107, 2003.

GODINHO FILHO, M.; FERNANDES, F. C. F.; LIMA, A. D. Pesquisa em Gestão da Produção na indústria de calçados: revisão, classificação e análise. Gestão e Produção, v. 16, n. 2, p.163-186, 2009.

GÓMEZ, R. C.; VIDAL, J. A.; ALCAMÍ, R. L. A model of product design management in the Spanish ceramic sector. European Journal of Innovation Management, v. 7, n. 2, p.150-161, 2004.

GONZÁLEZ, F. J. M.; PALACIOS T. M. B. The effect of new product development techniques on new product success in Spanish firms. Industrial Marketing Management, v. 31, p.261-271, 2002. 
GRIFFIN, A. PDMA research on new product development practices: Updating trends and benchmarking best practices. Journal of Product Innovation Management, v. 14, p.429-458, 1997.

HUNT, I.; JONES, R. Winning new product business in the contract electronics industry. International Journal of Operations \& Production Management, v. 18, n. 2, p.130142, 1998.

ILORI, M.O.; OKE, J. S.; SANNI, S. A. Management of new product development in selected food companies in Nigeria. Technovation, v. 20, p.333-342, 2000.

JUN, HONG-BAE; SUH, HYO-WON. A modeling framework for product development process considering its characteristics. IEEE Transactions on engineering management, v. 55, n. 1, 2008.

KOUFTEROS, X.; VONDEREMBSE, M.; JAYARAM, J. Internal and external integration for product development: the contingency effects of uncertainty, equivocality, and platform strategy. Decision Sciences, v.36, n.1, 2005.

LEDWITH, A. Management of new product development in small electronics firms. Journal of European Industrial Training, v.24, n.2, p.137-148, 2000.

LEE, J.; LEE, J.; SONDER, W. E. Differences of organizational characteristics in new product development: Crosscultural comparison of Korea and the US. Technovation, v. 20, n.9, p.497-508, 2000.

LIM, J. S.; SHARKEY, T. W.; HEINRICHS, J. H. New product development practices and export involvement: an initial inquiry. International Journal of Innovation Management, v. 7, n. 4, p.475-499, 2003.

LOCH, C.; STEIN, L.; TERWIESCH, C. Measuring product development performance in the electronics industry. Journal of Product Innovation Management, v. 13, p.3-20, 1996.

MACGREGOR, S. P.; ARANA, J.; PARRA, I.; LORENZO, M. P. Supporting new product creation in the Mondrago'n Valley. European Journal of Innovation Management, $v$. 9, n. 4, p.418-443, 2006

MALUF FILHO, W. M. Modelo para gestão do desenvolvimento e produção de pneus fornecidos para indústria automobilística. Tese (Doutorado) - Universidade de São Paulo, Departamento de Engenharia Mecânica, São Paulo/SP, 2008. 
MARCH-CHORDA, I.; GUNASEKARAN, A.; LLORIA-ARAMBURO, B. Product development process in spanish SMEs: an empirical research. Technovation, v. 22, p.301-312, 2002.

MARION, T. J. ; SIMPSON, T. W. New product development practice application to an early-stage firm: the case of the PaperPro-StackMaster. Design Studies, v. 30, n. 5, 2009.

MCIVOR, R.; HUMPHREYS, P. Early supplier involvement in the design process: lessons from the electronics industry. Omega, v. 32, p.179-199, 2004.

MELLO, C. H. P. Modelo para projeto e desenvolvimento de serviços. Tese (Doutorado) - Universidade de São Paulo. São Paulo/SP, 2005.

MENDES, G. H. S. O processo de desenvolvimento de produtos de empresas de base tecnológica: caracterização da gestão e proposta de modelo de referência. Tese (Doutorado) - Universidade Federal de São Carlos. São Carlos/SP, 2008.

MIGUEL, P. A. C. Estudo de caso na engenharia de produção: estruturação e recomendações para sua condução. Produção, v. 17, n.1, p.216-229, 2007.

MILLER, B.; CLARKE, J. P. Strategic guidance in the development of new aircraft programs: a practical real options approach. Transactions on Engineering Management, v. 55, n. 4, p.566-578, 2008.

MILLWARD, H.; BYRNE, C.; WALTERS, A.; LEWIS, A. New product development within small and medium-sized enterprises: analysis through technology management maps. International Journal of Innovation and Technology Management, v. 3, n. 3, p.283-302, 2006.

MONTEMEZZO, M. C. F. S. Diretrizes metodológicas para o projeto de produtos de moda no âmbito acadêmico. Dissertação (Mestrado em Design Industrial)Universidade Estadual Paulista, Bauru/SP, 2003.

NADIA, B.; GREGORY, G.; VINCE, T. Engineering change request management in a new product development process. European Journal of Innovation Management, $v$. 9, n. 1, p. 5-19, 2006.

NETO, J. P. B.; NOBRE, J. A. P. O processo de desenvolvimento de produto imobiliário: estudo exploratório em uma incorporadora. Produção, v. 19, n.1, p. 87-104, 2009.

NIJSSEN, E. J.; FRAMBACH, R. T. Determinants of the adoption of new product development tools by industrial firms. Industrial Marketing Management, v. 29, n. 2, p.121-131, 2000. 
NWABUEZE, U.; LAW, Z. C. The journey for survival: the case of new product development in the brewery industry. Journal of Product \& Brand Management, v. 10, n. 6, p. 382-397, 2001.

OGLIARI, A. Sistematização da concepção de produtos auxiliada por computador com aplicações no domínio de componentes de plástico injetado. Tese (Doutorado em Engenharia Mecânica) - Universidade Federal de Santa Catarina. Florianópolis/SC, 1999.

OTTENBACHER, M.; HARRINGTON, R. J. The innovation development process of Michelin-starred chefs. International Journal of Contemporary Hospitality Management, v. 19, n. 6, p. 444-460, 2007.

PAHL, G.; BEITZ,W.; FELDHUSEN, J.; GROTE, K. Projeto na engenharia:

fundamentos do desenvolvimento eficaz de produtos: métodos e aplicações. São Paulo: Edgard Blücher, 2005.

PAULA, I. C.; RIBEIRO, J. L. D. A proposal of a reference model for the pharmaceutical PDP management. Brazilian Journal of Operations \& Production Management, v. 4, n. 2, p.5-32, 2007.

PAULA, R. A. S. R.; CHENG, L. C. A transformação dos resultados de pesquisas científicas em novos produtos de base tecnológica, compreendida a partir do estudo de caso exploratório de projetos apoiados pela primeira experiência do Sebraetec na UFMG. CONGRESSO BRASILEIRO DE GESTÃO DE DESENVOLVIMENTO DE PRODUTO. 5. 2005. Anais. Curitiba/PR, 2005.

PETERS, A. J.; ROONEY, E. M.; ROGERSON, J. H.; MCQUATER, R. E.; SPRING, M.; DALE, B. G. New product design and development: a generic model. The TQM Magazine, v. 11, n. 3, p.172-179, 1999.

PRASNIKAR, J.; SKERLJ, T. New product development process and time-to-market in the generic pharmaceutical industry. Industrial Marketing Management, v. 35, p.690702, 2006.

RAMPERSAD, H. K. Concurrent design of product, process and robotic assembly system. Assembly Automation, v. 15, n. 1, p.21-28, 1995.

ROMANO, L. N. Modelo de referência para o processo de desenvolvimento de máquinas agrícolas. Tese (Doutorado) - Universidade Federal de Santa Catarina. Florianópolis/SC, 2003. 
ROSENTHAL, S. R. Effective Product Design and Development - How to cut lead time and increase customer satisfaction. New York, N.Y. Irwin Professional Publishing, 1992.

ROZENFELD, H.; FORCELLINI, F. A.; AMARAL, D. C.; TOLEDO, J. C.; SILVA, S. L.; ALLIPRANDINI, D. H.; SCALICE, R. K. Gestão de desenvolvimento de produtos: uma referência para a melhoria do processo. São Paulo: Editora Saraiva, 2006.

RUDOLPH, M. J. The food product development process. British Food Journal, v. 97, n. 3, p.3-11, 1995.

RUNDQUIST, J.; CHIBBA, A. The use of processes and methods in npd: a survey of swedish industry. International Journal of Innovation and Technology Management, v. 1, n. 1, p.37-54, 2004.

SCHMIDT, J. B.; SARANGEE, K. R., MONTOYA, M. M. Exploring new product development project review practices. Journal of Product Innovation Management, $\mathrm{v}$. 26, n. 5, p. 520-535, 2009.

SILVA, C. E. S. Método de avaliação do desempenho do processo de desenvolvimento de produtos. Tese (Doutorado em Engenharia de Produção) Universidade Federal de Santa Catarina. Florianópolis/SC, 2001.

SMITH, R. P.; MORROW, J. A. Product development process modeling. Design Studies, v. 20, p. 237-261, 1999.

SPRING, M.; MCQUATER, R.; SWIFT, K.; DALE, B.; BOOKER, J. The use of quality tools and techniques in product introduction: an assessment methodology. The TQM Magazine, v. 10, n. 1, p.45-50, 1998.

STALK, G. Time: the next source of competitive advantage. Harvard Business Review, v. 66 , n. 4, p.41-51, 1998.

STALK, G.; HOUT, T. M. Competing against time: how time-based competition is reshaping global markets. New York: The Free Press, 1990.

SUAREZ, T. M.; JUNG, C. F.; CATEN, C. S. Adaptação e aplicação de um método de desenvolvimento de produtos em uma microempresa de manufatura de produtos decorativos. Revista P\&D em Engenharia de Produção, v. 7, n. 1, p.37-63, 2009.

THIA, C. W.; CHAI, KAH-HIN; BAULY, J.; XIN, Y. An exploratory study of the use of quality tools and techniques in product development. The TQM Magazine, v. 17, n. 5, p.406-424, 2005. 
THIER, F. Modelo para o processo de desenvolvimento de máquinas para a indústria de cerâmica vermelha. Tese (Doutorado)- Universidade Federal de Santa Catarina, 2005.

TONI, A.; NASSIMBENI, G. Small and medium district enterprises and the new product development challenge: Evidence from Italian eyewear district. International Journal of Operations \& Production Management, v. 23, n. 6, p.678-697, 2003.

TRIM, P.; PAN, H. A new product launch strategy (NPLS) model for pharmaceutical companies. European Business Review, v. 17, n. 4, p.325-339, 2005.

VERNADAT, F. B. Enterprise modeling and integration: principles and applications. London: Chapman and Hall, 1996.

VINCENT, G. Managing new product development. New York:Van Nostrand Reinold, 1989.

WANG, L.; CHU, J.; WU, J. Selection of optimum maintenance strategies based on a fuzzy analytic hierarchy process. International Journal of Production Economics, v.107, n. 1. p.151-163, 2007.

WHEELWRIGHT, S. C.; CLARK, K. B. Revolutionizing product development: quantum leaps in speed, efficiency and quality. New York: Free Press, 1992.

XIN, J. Y.; YENG, A. C. L. E.; CHENG, T. C. E. Radical innovations in new product development and their financial performance implications: An event study of US manufacturing firms. Operations Management Research, v. 1, n. 2, p.119-128, 2008.

YEH, T. M.; PAI, F. Y.; YANG, C. C. Performance improvement in new product development with effective tools and techniques adoption for high-tech industries. Quality and Quantity, doi 10.1007/s11135-008-9186-7, 2008.

ZANCUL, E. S.; ROZENFELD, H. Modelo de referência do processo de desenvolvimento de produtos populares. Congresso Brasileiro de Gestão de Desenvolvimento de Produto. 5. 2005. Anais. Curitiba/PR, 2005.

ZANCUL, E. S.; MARX, R.; METZKER, A. Organização do trabalho no processo de desenvolvimento de produtos: a aplicação da engenharia simultânea em duas montadoras de veículos. Gestão e Produção, v.13, n.1, p. 15-29, 2006.

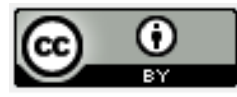

Artigo recebido em 14/12/2009 e aceito para publicação em 03/10/2010. 